\title{
Clinical Dilemma: Recurrent Syncope With Isolated Left Ventricle Non-Compaction Cardiomyopathy and Preserved Ejection Fraction, a Case Report and Review of the Literature
}

\author{
Ali Ridha ${ }^{\mathrm{a}, \mathrm{c}}$, Adnan Khan ${ }^{\mathrm{a}}$, Sarah Al-Abayechi ${ }^{\mathrm{b}}$, Eric Yeung ${ }^{\mathrm{a}}$
}

\begin{abstract}
Left ventricle non-compaction cardiomyopathy (LVNC) is an uncommon disorder characterized by the persistence of fetal myocardium of prominent trabecular meshwork, deep intertrabecular recesses, systolic dysfunction and left ventricular dilatation. It is thought to be caused by the arrest of normal endomyocardial morphogenesis. Here we present a case of a 27 -year-old Caucasian female without past medical history who was presented to the emergency department because of syncope while she was working out. On further questioning, she relates that she has been having recurrent syncope for the last 9 years but she did not seek medical attention. Echocardiography and cardiac magnetic resonance imaging confirmed the diagnosis of LVNC and also showed a preserved ejection fraction. Implantable loop recorder showed recurrent non-sustained ventricular tachycardia. Although data were limited, an implantable cardioverter-defibrillator was prophylactically implanted because of the individual's high sudden cardiac death risk.
\end{abstract}

Keywords: Non-compaction; Cardiomyopathy; Hypertrabeculation; Implantable cardioverter-defibrillator

\section{Introduction}

Non-compaction cardiomyopathy of the ventricles is a rare genetic cardiomyopathy. It has been suggested that it may be due to intrauterine arrest of compaction of the loose interwoven meshwork that makes up the fetal myocardial primordium. Usually the left ventricle is the most commonly reported affected site, but few cases of right ventricular involvement have also been reported. The major clinical manifestations of noncompaction cardiomyopathy are heart failure, arrhythmias, and

Manuscript accepted for publication January 05, 2015

aRosalind Franklin University, 3333 Green Bay Rd, North Chicago, IL 60064, USA

${ }^{\mathrm{b} C o l l e g e}$ of Medicine/Baghdad University, Baghdad, Iraq

${ }^{c}$ Corresponding Author: Ali Ridha, Rosalind Franklin University, 3333 Green Bay Rd, North Chicago, IL 60064, USA. Email: alim.ridha@yahoo.com

doi: http://dx.doi.org/10.14740/jmc2035w embolic events. Echocardiography and/or cardiac magnetic resonance imaging is considered the reference standard for the diagnosis of non-compaction cardiomyopathy. Outcomes and appropriate therapies remain poorly defined. Pharmacological treatment and implantable cardioverter-defibrillator (ICD) may be considered for the management of ventricular arrhythmias in patients with this cardiomyopathy. Here, we present a case where left ventricle non-compaction cardiomyopathy (LVNC) with preserved ejection fraction of recurrent syncope is diagnosed in a 27-year-old treated with ICD.

\section{Case Report}

A 27-year-old female was presented to the emergency department with syncope while she was working out. The syncope was associated with chest tightness, and palpitation. The patient relates that she had several episodes of syncope and nearsyncope for the last 9 years. Usually, the syncope episodes were led by palpitations and lightheadedness. There was no history of chest pain, hypertension, or diabetes mellitus. There is no family history of cardiovascular disease or sudden cardiac death (SCD). The patient has been told in the past to be vasovagal syncope. Physical exam was unremarkable with a blood pressure of $114 / 63 \mathrm{~mm} \mathrm{Hg}$ and pulse rate of $84 / \mathrm{min}$. Laboratory investigations showed hemoglobin was $14 \mathrm{~g} / \mathrm{dL}$. The total WBC count was 7,800 with normal differentiation. Liver and renal functions were within normal limits. Serum electrolytes (sodium, calcium, potassium and magnesium) were within normal range. Electrocardiogram (ECG) showed sinus rhythm with occasional premature ventricular complexes. The patient was referred to the Cardiology Department for further evaluation.

As part of cardiology workup, two-dimensional transthoracic echocardiography (TTE) demonstrated normal left ventricle ejection fraction (LVEF), no regional wall motion abnormality, there is hypertrabeculation of apex and inferolateral walls best seen in short axis with contrast flowing between the trabeculi and ratio of compacted to non-compacted myocardium $>2: 1$ in end systolic frame (parasternal short axis) concerned for LVNC (Fig. 1). The patient had a follow-up cardiac MRI which confirmed the diagnosis of LVNC (Fig. 2). The patient had a stress echocardiogram which was negative for ischemia. Genetic testing showed DES c.635 GA p. Arg- 


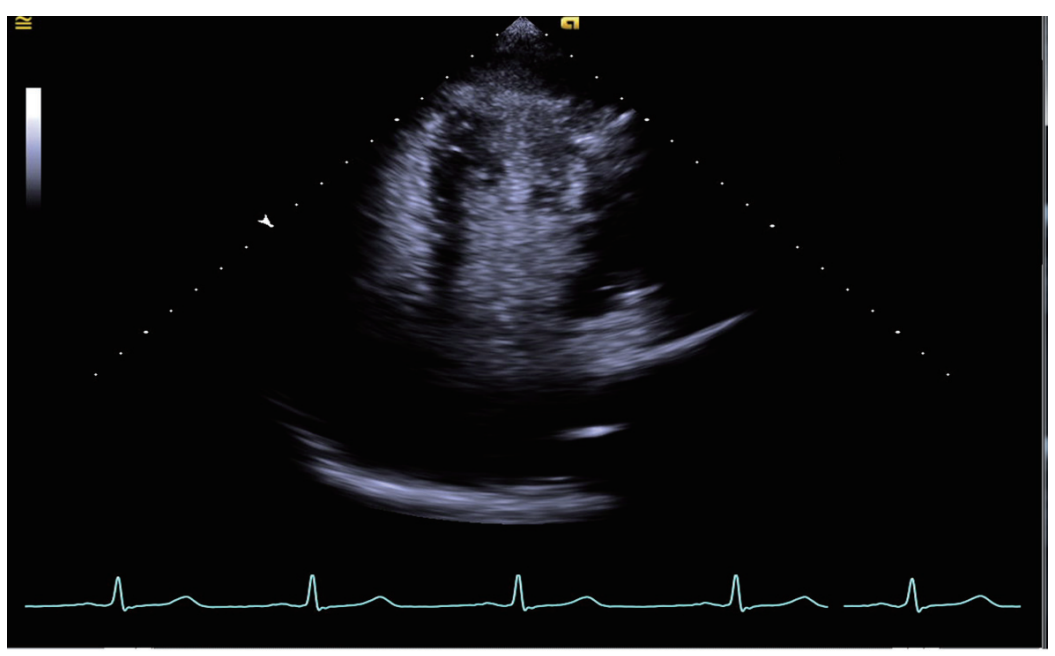

Figure 1. Heavy trabeculation of the left ventricular apex, distal septal, and lateral walls.

212GLn, heterozygous variant of unknown significance. The patient was started on beta blocker and implantable loop recorder placed which showed only PVC and eight beat runs of recurrent non-sustained ventricular tachycardia (NSVT) that is associated with palpitations but no ventricular tachycardia (VT). The patient was considered at a higher risk since she lives alone, NSVT, LVNC, and had a history of recurrent syncope; therefore, eventually the patient was supplied with ICD single lead single chamber. She was asked to follow up with TTE yearly. The family members were contacted and advised for genetic screening.

\section{Discussion}

Non-compaction cardiomyopathy is one of rarest congenital diseases that involve the myocardium of the ventricles [1].

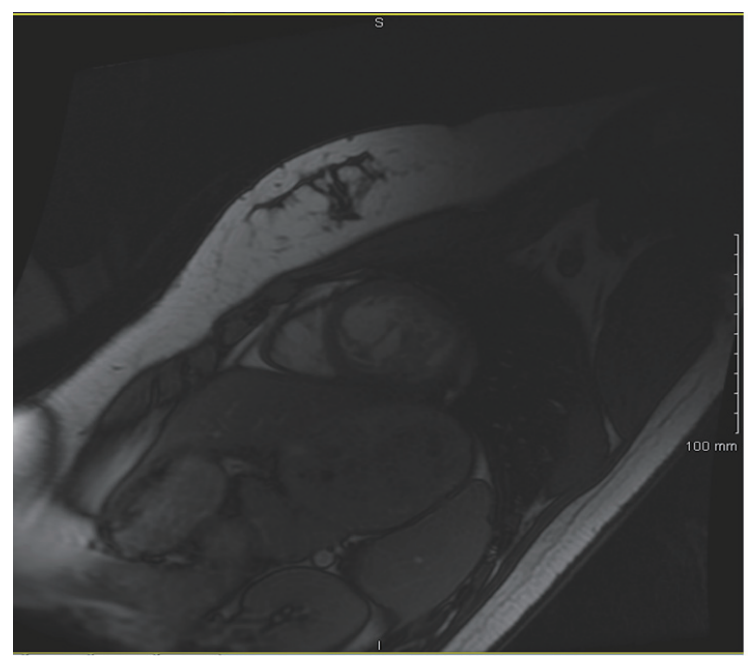

Figure 2. Cardiac magnetic resonance imaging in short axis T1 FLAIR shows a left ventricle with non-compacted to compacted myocardium to $2.9: 1$.
The prevalence is estimated at $0.014-1.3 \%$ [2]. It is defined by intrauterine developmental arrest of the normal compaction process of the myocardium during the first trimester leading to the formation of two layers of the myocardium: the compacted and the non-compacted layer [3]. Usually it is commonly observed in the left ventricle but the right ventricle can also be affected [4]. The main clinical appearances of LVNC are heart failure $25 \%$, atrial and ventricular arrhythmias $10-26 \%$, thromboembolic events, and SCD [5]. Some patients may present with palpitation, chest pain shortness of breath, syncope, an abnormal heart sound, or an abnormal electrocardiogram or echocardiogram [1]. Our patient presented with syncope that usually proceeded by palpitation.

The condition is diagnosed by two-dimensional echocardiography and/or magnetic resonance imaging, viewing large prominent trabeculations and deep intertrabecular recesses, in which flow can be detected. Furthermore, the two-layered wall structure (thin compacted epicardium and thick, noncompacted endocardium) is essential for the diagnosis [4]. In our patient the TTE was highly suggestive of LVNC by Jenni et al criteria [6]. The direct blood flow was from the ventricular cavity into the deep intertrabecular recesses, as seen by color Doppler echocardiography with six prominent trabeculae [7].

There is no precise therapy for LVNC. Medical management should target the clinical manifestations: LVEF, the presence or absence of arrhythmias, and perceived risk of thromboembolism. For the high risk of atrial and ventricular arrhythmias, the better strategy is to monitor patients with periodic Holters to detect asymptomatic arrhythmias [8]. Expanded monitoring by a loop recorder might also provide better information [9]. The role of electrophysiological study (EPS) to determine the risk for ventricular arrhythmia and SCD is not well established in LVNC. ICD implantation is one of the treatment options but its superiority over medical therapy is still being debated and there are limited data on long-term follow-up [10].

Kobza et al conducted a retrospective study on 12 patients with LVNC during a median follow-up of 36 months. Patients underwent ICD implantation for primary and secondary pre- 
vention since potentially life-threatening ventricular tachyarrhythmias may occur in patients with LVNC, and only five patients received appropriate ICD therapy. They also conducted supraventricular tachyarrhythmias devices with reliable detection enhancements which should be considered [11]. Likewise, Celiker et al reported a 6-year-old child with LVNC and ICD who had three ventricular fibrillation episodes which were treated with the device by appropriate shocks during a followup of 16 months [12]. Duru et al reported a patient with LVNC and ICD who had developed numerous different VT episodes that were appropriately treated by the device [13].

In contrast, Stanton et al analyzed the follow-up data of 30 patients with LVNC during 2.5 years and reported no appropriate therapies among the 11 LVNC patients with ICD. Also during the follow-up they did not find any difference in mortality between patients with LVNC and patients with dilated cardiomyopathy. They stated that the necessity of ICD implantation is being argued, and nearly all reports have pointed out that supraventricular tachyarrhythmias, especially new onset atrial fibrillation, might lead to inappropriate ICD discharges. While the deaths in the LVNC group (three patients out of 30) were observed only in patients with decreased LVEF, suggesting that ICD therapy might be reserved for this subgroup [2].

Since there are no clear cut guidelines for ICD implantation for patients with LVNC, a number of writers favor to implant an ICD even if NSVT or fibrillation is observed during EPS [9]. Other authors stated that symptoms of heart failure, a history of sustained VT or an enlarged left atrium were associated with an unstable course and more severe prognosis [14]. Oechslin et al stated that certain clinical characteristics were more frequently observed in non-survivors compared with survivors with LVNC, including higher LV end-diastolic diameter on presentation, NYHA class III-IV, permanent or persistent atrial fibrillation, and bundle branch block. Patients with these high risk features might be candidates for early, aggressive intervention, including consideration of ICD implantation and evaluation for transplantation [1].

There is a lack of risk stratification model for patients with LVNC for preserved ejection fraction. In our case, we considered our patient to be at high risk and supplied her with an ICD since we suspected her syncopal events to be secondary to ventricular tachycardia, with only evidence of NSVT on monitoring. ICD will provide both a monitoring tool, as well as a therapeutic tool for patients like our own, in which her clinical future is uncertain.

\section{Conclusion}

Non-compaction cardiomyopathy is a rare primary genetic disorder, with prevalence of $0.014-1.3 \%$. Misdiagnosis/late diagnosis is very common, as clinical manifestations vary from heart failure, arrhythmia, syncope and thromboembolic events. As in our case non-compaction cardiomyopathy should be considered in the deferential diagnosis of recurrent syncope, though we have limited data to make a firm conclusion on the prognosis of these patients. The best therapeutic decision should be based on the patient's own clinical features and the physician's judgment. We recommend ICD in cases of aborted cardiac arrest, sustained VT, syncope related to VA, family history of SCD, or severely impaired LVEF.

\section{References}

1. Oechslin EN, Attenhofer Jost CH, Rojas JR, Kaufmann PA, Jenni R. Long-term follow-up of 34 adults with isolated left ventricular noncompaction: a distinct cardiomyopathy with poor prognosis. J Am Coll Cardiol. 2000;36(2):493-500.

2. Stanton C, Bruce C, Connolly H, Brady P, Syed I, Hodge $\mathrm{D}$, Asirvatham $\mathrm{S}$, et al. Isolated left ventricular noncompaction syndrome. Am J Cardiol. 2009;104(8):11351138.

3. Weiford BC, Subbarao VD, Mulhern KM. Noncompaction of the ventricular myocardium. Circulation. 2004;109(24):2965-2971.

4. Maron BJ, Towbin JA, Thiene G, Antzelevitch C, Corrado D, Arnett D, Moss AJ, et al. Contemporary definitions and classification of the cardiomyopathies: an American Heart Association Scientific Statement from the Council on Clinical Cardiology, Heart Failure and Transplantation Committee; Quality of Care and Outcomes Research and Functional Genomics and Translational Biology Interdisciplinary Working Groups; and Council on Epidemiology and Prevention. Circulation. 2006;113(14):1807-1816.

5. Murphy RT, Thaman R, Blanes JG, Ward D, Sevdalis E, Papra E, Kiotsekoglou A, et al. Natural history and familial characteristics of isolated left ventricular non-compaction. Eur Heart J. 2005;26(2):187-192.

6. Jenni R, Oechslin E, Schneider J, Attenhofer Jost C, Kaufmann PA. Echocardiographic and pathoanatomical characteristics of isolated left ventricular non-compaction: a step towards classification as a distinct cardiomyopathy. Heart. 2001;86(6):666-671.

7. Espinola-Zavaleta N, Soto ME, Castellanos LM, JativaChavez S, Keirns C. Non-compacted cardiomyopathy: clinical-echocardiographic study. Cardiovasc Ultrasound. 2006;4:35.

8. Finsterer J, Stollberger C. Consensus on unsolved issues of left ventricular hypertrabeculation/noncompaction is warranted. Int J Cardiol. 2010;145(3):497; author reply 498-499.

9. Coppola G, Guttilla D, Corrado E, Falletta C, Marrone G, Farulla RA, Ciaramitaro G, et al. ICD implantation in noncompaction of the left ventricular myocardium: a case report. Pacing Clin Electrophysiol. 2009;32(8):10921095.

10. Jenni R, Oechslin EN, van der Loo B. Isolated ventricular non-compaction of the myocardium in adults. Heart. 2007;93(1):11-15.

11. Kobza R, Jenni R, Erne P, Oechslin E, Duru F. Implantable cardioverter-defibrillators in patients with left ventricular noncompaction. Pacing Clin Electrophysiol. 2008;31(4):461-467.

12. Celiker A, Kafali G, Dogan R. Cardioverter defibrillator 
implantation in a child with isolated noncompaction of the ventricular myocardium and ventricular fibrillation. Pacing Clin Electrophysiol. 2004;27(1):104-108.

13. Duru F, Candinas R. Noncompaction of ventricular myocardium and arrhythmias. J Cardiovasc Electrophysiol.
2000;11(4):493.

14. Lofiego C, Biagini E, Pasquale F, Ferlito M, Rocchi G, Perugini E, Bacchi-Reggiani L, et al. Wide spectrum of presentation and variable outcomes of isolated left ventricular non-compaction. Heart. 2007;93(1):65-71. 